\section{Multichannel ECG Data Compression by Multirate Signal Processing and Transform Domain Coding Techniques}

\author{
A. Enis Cetin, Hayrettin Köymen, and M. Cengiz Aydın
}

\begin{abstract}
In this paper, a multilead ECG data compression metho is presented. First, a linear transform is applied to the standard ECG lead signals which are highly correlated with each other. In this way a set of uncorrelated transform domain signals is obtained. Then, resulting transform domain signals are compressed using various coding methods, including multirate signal processing and transform domain coding techniques.
\end{abstract}

\section{INTRODUCTION}

Computerized electrocardigram (ECG) processing systems have been widely used in clinical practice [1]. They are capable of processing long records of ECG's. The amount of data generated by these devices becomes excessive very quickly. Data compression techniques which have been successfully utilized in speech and video signals [2] can be utilized in many practical applications including: i) computerized ECG data bases, ii) ambulatory recording systems such as digital Holter recorders, and iii) ECG codecs which transmit ECG signals over digital telecommunication networks.

An extensive review of ECG data compression techniques was given in [3]. The aim of any ECG data compression scheme is to achieve maximum data volume reduction without loosing clinically significant information [3], [4]. This paper presents a new multilead ECG data compression technique. In this technique samples of the standard ECG lead signals are first linearly transformed. Then, resulting transform domain signals are compressed using various coding methods, including multirate signal processing and transform coding methods.

\section{Multichannel Compression Procedure}

In this section, the multichannel compression method whose block diagram is shown in Fig. 1 is described.

Twelve ECG signals are first passed through a preprocessor. The function of the preprocessor is to prepare raw ECG data for further processing. After preprocessing the input signals, the resulting discrete-time sequences are linearly tansformed into another set of sequences. The aim of this linear transformation is to decorrelate the highly correlated ECG lead signals. The transformation matrix, $A$, can he the matrix of the optimum transform, Karhunen-Loève transform (KLT), or the matrix of a suboptimum transform such as the discrete cosine transform (DCT). Lastly, to compress the transform domain signals various coding schemes which exploit their special nature are utilized.

In the following subsections, detailed descriptions of the sub-blocks of the multichannel ECG compression method are given.

\section{A. The Preprocessor}

In this paper, the standard twelve-channel ECG lead system is considered. This ECG recording configuration consists of twelve ECG

Manuscript received September 24, 1991; revised October 16, 1992. This work was supported by TUBITAK (Scientific and Technical Research Council of Turkey).

The authors are with the Deparment of Electrical and Electronics Engineering, Bilkent University, Bilkent, Ankara 06533, Turkey.

IEEE Log Number 9207668 leads, I, II, II, AVR, AVL, AVF, V1, V2, . ., and V6. The leads, III, AVR, AVL, and AVF, are linearly related to I and II. Therefore only eight channels are enough to represent standard twelve channel ECG recording system.

The preprocessor discards the redundant channels, III, AVR, AVL and AVF, and rearranges the order of the ECG channels in order to bring correlated channels close to each other. The six precordial (chest) leads, V1, $\cdots, \mathrm{V} 6$, represent variations of the electrical heart vector amplitude with respect to time from six different narrow angles. During a cardiac cycle it is natural to expect high correlation among precordial leads so the channels $\mathrm{V} 1, \cdots, \mathrm{V} 6$ are selected as the first 6 signals, i.e., $x_{i-1}=V i, i=1,2, \cdots, 6$. The two horizontal lead waveforms (I and II) which have relatively less energy contents with respect to precordial ECG lead waveforms are chosen as seventh, $x_{6}=\mathrm{I}$, and eighth channels, $x_{7}=\mathrm{II}$. A typical set of standard ECG lead waveforms, $x_{i}, i=0,1, \cdots, 7$, are shown in Fig. 3 .

The aim of the reordering the ECG channels is to increase the efficiency of the linear transformation operation which is described in the next

\section{B. The Linear Transformer}

The outputs of the preprocessor block, $x_{i}, i=0,1, \cdots, 7$, are fed to the linear transformer. In this paper, both the optimum transform, Karhunen-Loève, and a suboptimum transform, DCT are used.

In this block, the ECG channels are linearly transformed to another domain, and 8 new transform domain signals $y_{i}, i=0,1, \cdots, 7$, which are significantly less correlated (ideally uncorrelated) than the ECG signal set, $x_{i}, i=0,1, \cdots, 7$, are obtained.

Let $x_{k}(m), k=0,1, \cdots, N-1$ ( $N$ is equal to eight in our case), be the reordered ECG signal samples at discrete time instant $m$, the transform domain samples at time instant $m$ are given as follows:

$$
Y_{m}=A \cdot X_{m}
$$

where $Y_{m}=\left[y_{0}(m), \cdots, y_{N-1}(m)\right]^{T}, X_{m}=\left[x_{0}(m), \cdots\right.$, $\left.x_{N-1}(m)\right]^{T}$, and $A$ is the $N \times N$ transform matrix.

The optimum linear transform, discrete Karhunen-Loève ransform (KLT), can be properly defined for stationary random processes and the entries of the transform matrix, $A_{\mathrm{KLT}}$ depend on the statistics of the random processes. For slowly varying unstationary signals an approximate KLT matrix can also be defined. Although ECG signals cannot be considered to be wide sense stationary random processes, a covariance matrix, $\hat{C}_{x}$, of the ECG channels is estimated as follows:

$$
\hat{C}_{X}=\frac{1}{M} \sum_{i=0}^{M-1}\left[\begin{array}{c}
x_{0}(i) \\
\vdots \\
x_{N-1}(i)
\end{array}\right]\left[x_{0}(i) \cdots x_{N-1}(i)\right]
$$

where $N$ is the number of the ECG channels and $M$ is the number of ECG samples per channel used. The $N \times N$ ECG channel covariance matrix, $\hat{C}_{x}$, is used in the construction of an approximate KLT matrix. Rows of the approximate KLT matrix are the eigenvectors of $\hat{C}_{x}$. The $8 \times 8$ KLT matrix shown in (3) at the bottom of the next page is obtained by using 1024 ECG samples per channel.

There is no fast algorithm to compute the KL transform. In this case, the computational burden is not high because $N$ is only equal to 8 .

The discrete cosine transform (DCT) is also used as a linear transformer. The DCT matrix approximates KLT matrix in the case of 


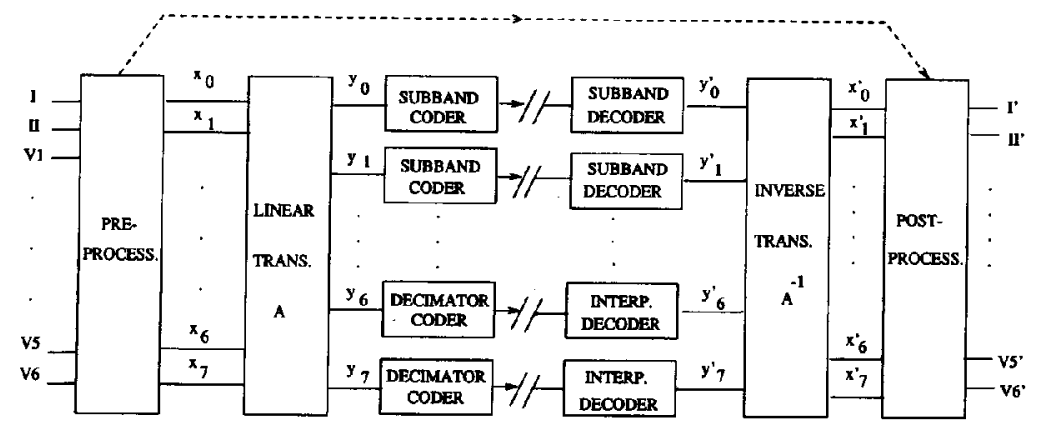

Fig. 1. Block diagram of the multichannel ECG data compression scheme.

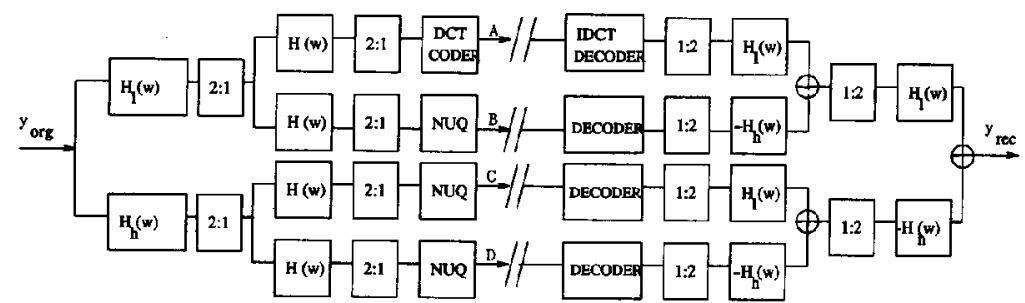

Fig. 2. Subband (SB) coding structure which is utilized to compress the high energy signals, $y_{i}, i=0,1, \cdots, 4$. (DCT: Discrete Cosine Transform, IDCT: Inverse DCT, 2 : 1 Downsampler by a factor of $2,1: 2$ Upsampler by a factor of 2 , NUQ: Nonuniform Quantizer)

high correlation [2], [7] among input signals. The DCT of a sequence $v(n), n=0,1, \cdots, N-1$, is defined as

$G(k)=\sqrt{\frac{\alpha_{k}}{N}} \sum_{n=0}^{N-1} v(n) \operatorname{Cos} \frac{(2 n+1) k \pi}{2 N} \quad k=0,1,2, \cdots, N-1$

where $G(k)$ is the $k$ th DCT coefficient, and $\alpha_{k}=1$ for $k=0$ and $\alpha_{k}=2$ for other $k$ values.

There exist fast algorithms, order $(N \log N)$, to compute the DCT [7]. Therefore it is computationally more efficient to implement the DCT than the KLT which requires order $\left(N^{2}\right)$ multiplications.

\section{Compression of the Transform Domain Signals}

In this section the compression of the uncorrelated transform domain signals $y_{k}, k=0,1, \cdots, 7$ is described. In Fig. 4 a typical set of uncorrelated signals, $y_{k}, k=0,1, \cdots, 7$, are shown. The signals in Fig. 4 are obtained by $\mathrm{KL}$ transforming the ECG signals, $x_{k}, k=0,1, \cdots, 7$, shown in Fig. 3 .
Transform domain signals, $y_{k}, k=0,1, \cdots, 7$, are divided into two classes according to their energy contents. The first class of signals, $y_{0}, y_{1}, \cdots, y_{4}$, have higher energy than the second class of signals, $y_{5}, y_{6}$, and $y_{7}$. High energy signals, $y_{0}, y_{1}, \cdots, y_{4}$, are faithfully compressed by using a subband (SB) coder [5]. Low energy signals, $y_{5}, y_{6}$, and $y_{7}$, have not only significantly less energy than the high energy signals but carry little information, as well. Low energy signals $y_{5}, y_{6}$, and $y_{7}$, are compressed by the multirate transform coder (MTC) structure. The description of the compression methods are given in the following subsections.

1) Subband (SB) Coder: High energy signals, $y_{0}(n), y_{1}(n), \cdots$, $y_{4}(n)$, contain more information than the low energy signals $y_{5}(n), y_{6}(n)$ and $y_{7}(n)$. Therefore the high energy signals, $y_{0}(n), \cdots, y_{4}(n)$ should be compressed very accurately. In [5], a SB coder for ECG signals is developed. In this SB coding scheme reconstructed ECG signals are visually almost indistinguishable from the original signals. Hence, in this paper, the use of this SB coder is decided to compress the signals, $y_{0}(n), \cdots, y_{4}(n)$.

The block diagram of the SB coder is shown in Fig. 2. The input signal is decomposed into four subsignals by using a quadrature

$$
A_{\text {KLT }}=\left[\begin{array}{rrrrrrrr}
0.1883 & 0.1568 & 0.4199 & 0.4874 & 0.5024 & 0.4509 & 0.2615 & 0.0452 \\
-0.1779 & -0.1707 & -0.3426 & -0.2350 & -0.0552 & 0.3290 & 0.6821 & 0.4360 \\
0.3715 & 0.5210 & 0.0591 & -0.1347 & -0.3928 & -0.0400 & 0.4749 & -0.4331 \\
-0.2164 & 0.7699 & -0.4322 & 0.1633 & 0.0982 & 0.0392 & -0.2231 & 0.2934 \\
-0.8073 & 0.0066 & 0.0553 & 0.1945 & -0.0723 & 0.1165 & 0.1529 & -0.5149 \\
-0.2528 & 0.2391 & 0.4301 & -0.4758 & 0.4233 & -0.4700 & 0.2042 & 0.1609 \\
-0.1722 & 0.0805 & 0.5204 & 0.2431 & -0.6256 & -0.0420 & -0.0233 & 0.4902 \\
0.0522 & -0.1359 & -0.2392 & 0.5823 & 0.0699 & -0.6702 & 0.3543 & 0.0544
\end{array}\right]
$$




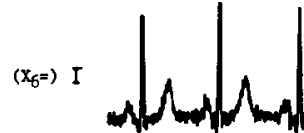

$\left(x_{2}=v^{3}\right)$

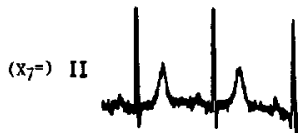<smiles>CC(C)CC(C)CC(C)CC(C)C</smiles><smiles>CC(C)CC(C)CC(C)C</smiles>
$\left(x_{4} \Rightarrow\right.$ V 5<smiles>CCC(C)CC(C)CC(C)(C)C</smiles><smiles>CC(C)CC(C)(C)CC(C)(C)N</smiles>
$\left(\mathrm{x}_{5}=\right)$ VE

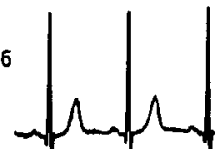

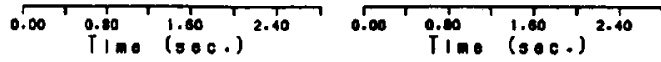

Fig. 3. A typical set of standard ECG lead waveforms, $x_{i}, i=0,1, \cdots, 7$.

mirror filter (QMF) bank in a tree structured manner. In the first stage of the tree the input signal, $y_{i}(i=0,1, \cdots, 4)$, is filtered by a lowpass filter $H_{l}$ and a highpass filter $H_{h}$. Outputs of these filters are downsampled by a factor of two. In this way the signal, $y_{i}$, is decomposed into two subsignals, $y_{i, 0}$ and $y_{i, 1}$. In the second stage of the tree, the subsignals, $y_{i, 0}$ and $y_{i, 1}$, are decomposed once again and four subsignals, $y_{i, j, k}, j=0,1 ; k=0,1$, whose sampling rates are four times lower than the sampling rate of the signal $y_{i}$ are obtained. The SB decomposition structure of Fig. 2 splits the signal $y_{i}$ into four consecutive bands, $[l \pi / 4,(l+1) \pi / 4], l=0,1,2,3$, in the frequency domain [2]. For example, $y_{i, 0,0}$ (the subsignal at branch $\mathrm{A}$ of Figure 2) comes from the low-pass frequency band, $[0 \pi / 4]$, of the signal $y_{i}$. In the coding of the subband signals, $y_{i, j, k}, j=0,1 ; k=0,1$, one takes the advantage of the nonuniform distribution of energy in the frequency domain to judiciously allocate the bits. The number of bits used to encode each frequency band can be different, so that the encoding accuracy is always maintained at the required frequency bands [2]. In the absence of quantization errors, a QMF bank based signal decomposition structure together with the corresponding signal reconstruction filter bank provides perfect reconstruction, i.e., $y_{\mathrm{rec}}(n)=y_{\text {org }}(n-K), K>0$, where $y_{\text {org }}(n)$ is the input and $y_{\mathrm{rec}}(n)$ is the output of the QMF bank. This is because of the fact [2] that the low pass, $H_{l}(\omega)$, and the high pass filter, $H_{h}(\omega)$ satisfy the following condition:

$$
\left|H_{l}(\omega)\right|^{2}+\left|H_{h}(\omega)\right|^{2}=1
$$

In other words filtering and downsampling operations in the SB decomposition structure shown in Fig. 2 do not introduce any loss.

It is observed that the energy of the signal $y_{i}$ is mainly concentrated

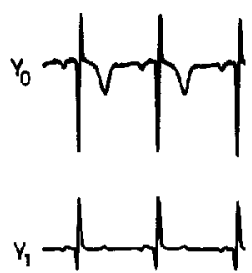

$\mathbf{Y}_{4}$

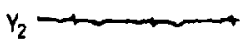

$Y_{6}$

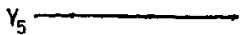

$Y_{3}$

$r_{7}$

Fig. 4. Uncorrelated signals, $y_{i}, i=0,1, \cdots, 7$, corresponding to the ECG signals shown in Fig. 3.

in the lowest frequency band $[0, \pi / 4]$. Because of this, the lowband subsignal $y_{i, 0,0}$ has to be carefully coded. High correlation among neighboring samples of $y_{i, 0,0}$ makes this signal a good candidate for efficient predictive or transform coding. Transform coding was also used in [8] to compress ECG signals. In this paper, the use of a DCT based scheme is also chosen to compress $y_{i, 0,0}$. After the application of DCT with a block size of 64 samples to the lowband subsignal, $y_{i, 0,0}$, the transform domain coefficients, $G_{i, 0,0}(k), k=0,1, \cdots, 63$ are obtained.

DCT coefficients, $G_{i, 0,0}(k)$, are thresholded and quantized. The coefficients whose magnitudes are above a preselected threshold, $\beta$, are retained and the other coefficients are discarded. Thresholding squeezes the dynamic range of the amplitude values. This operation is followed by the quantization of the thresholded DCT coefficients. Thresholded and quantized nonzero coefficients are variable length coded by using an amplitude table and the zero values are runlength coded. The amplitude and runlength lookup tables are Huffman coding tables which are obtained according to the histograms of the DCT coefficients.

In practice ECG recording levels do not change from one recording to another one. If drastic variations occur, one can first scale the input by a factor $\alpha$, then apply DCT.

Bandpass and highpass subsignals, $y_{i, 0,1}, y_{i, 1,0}$ and $y_{i, 1,1}$ (branches $B, C$, and $D$ ), are coded using non-uniform quantizers. After quantization, a code assignment procedure is realized using variable length amplitude and runlength lookup tables for zero values. The lookup tables are obtained according to the histograms of quantized subband signals. The above look-up tables and quantizers can be found in [6].

The bit streams which are obtained from coding of four subband signals are multiplexed and stored. Appropriate decoders are assigned to each branch to convert the bit streams into time domain samples and the four branch synthesis QMF bank performs the reconstruction [5].

2) Multirate Transform Coder (MTC) Structure: This subsection describes the coding of low energy signals, $y_{5}, y_{6}$ and $y_{7}$ which are compressed by the multirate transform coder (MTC) unit.

Low energy signals, $y_{5}(n), y_{6}(n)$ and $y_{7}(n)$, can be coded by using the SB coder described in Section II-C.1, too. However, it is observed that all the subsignals except the subsignal at branch 


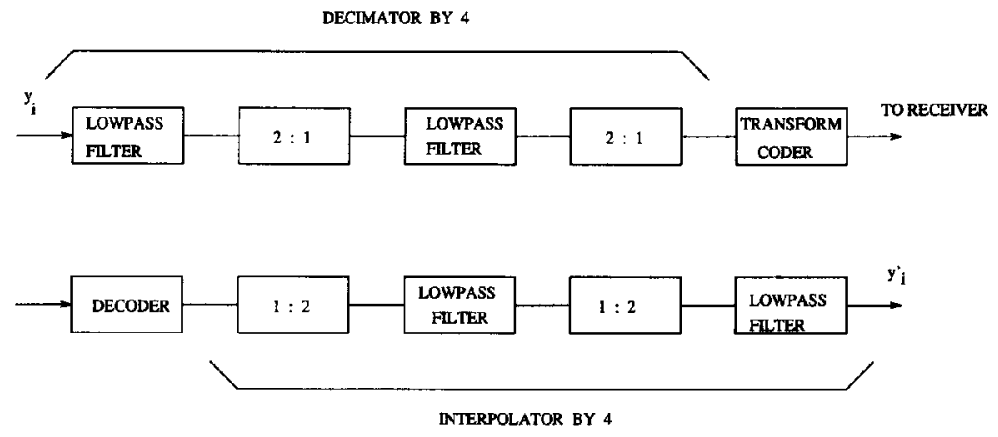

Fig. 5. Multirate Transform Coder (MTC) structure which is utilized to compress and decompress the low energy signals, $y_{i}, i=5,6,7$.

TABLE I

CR and APRD Values for the Multichannel ECG Signals Shown IN Fig. 3. For Each APRd Value Given in Column 4, Corresponding Coding Result for the Single-Channel SBC (Multichannel, by Using DCT) \{Multichannel by Using KLT\} Method is Grven IN Columa 1 (2) $\{3\}$.

\begin{tabular}{|c|c|c|c|}
\hline$C R$ of $S \bar{B} C$ & $C R(D C T)$ & $\bar{C} R(K L T)$ & $A P R D(\%)$ \\
\hline 4.02 & 4.97 & 6.19 & 3.70 \\
4.07 & 5.18 & 6.43 & 3.84 \\
4.19 & 5.22 & 6.62 & 4.04 \\
4.22 & 5.23 & 6.82 & 4.27 \\
4.31 & 5.54 & 7.09 & 4.57 \\
4.50 & 6.00 & 7.71 & 5.60 \\
4.57 & 6.08 & 7.87 & 5.93 \\
4.65 & 6.17 & 7.98 & 6.19 \\
\hline
\end{tabular}

$A$ of Fig. 2 contain very little information when QMF subband decomposition is applied to the signals $y_{5}(n), y_{6}(n)$, and $y_{7}(n)$. Due to this fact, only the subsignals at branch $A$ (low-band signals) are processed. The other branches are discarded. Although the three highfrequency branches of the SB coder are discarded it is experimentally observed that the reconstruction error for the signals, $y_{5}(n), y_{6}(n)$, and $y_{7}(n)$ is very close to the SB coding results.

Branch $A$ consists of a low-pass filter with a cutoff $\omega=\pi / 2$ and a downsampling by a factor of two block. In multirate signal processing this branch is called the decimator by a factor of two [9]. The downsampler by a factor of two performs a sampling rate reduction by dropping every other sample of its input sequence, i.e., $z(n)=w(2 n)$ where $w(n)$ and $z(n)$ is the input and output sequences of the downsampler, respectively. When a digital signal is downsampled, aliasing may occur. In order to avoid aliasing, the input is low-pass filtered before the downsampling operation. The seventhorder Lagrange filter is used as the antialiasing filter. The Lagrange half-band filter is the lowpass filter of the SB decomposition structure of $[10]$.

The block diagram of the MTC unit is shown in Fig. 5. In the MTC structure two successive blocks of decimators by a factor of two are used. In this way the sampling rate is reduced by a factor of four. After the decimation by a factor of four the signal, $y_{i}(n),(i=5,6,7)$, is compressed by the coder block. This coder is the same as the coder utilized in the branch $A$ of the SB coder.

Reconstruction of the transform domain signals begin with decoding the compressed bit stream. A tree-search is needed to convert variable length coded samples to DCT amplitude values. Inverse DCT is applied to transform these amplitude values to the time domain.
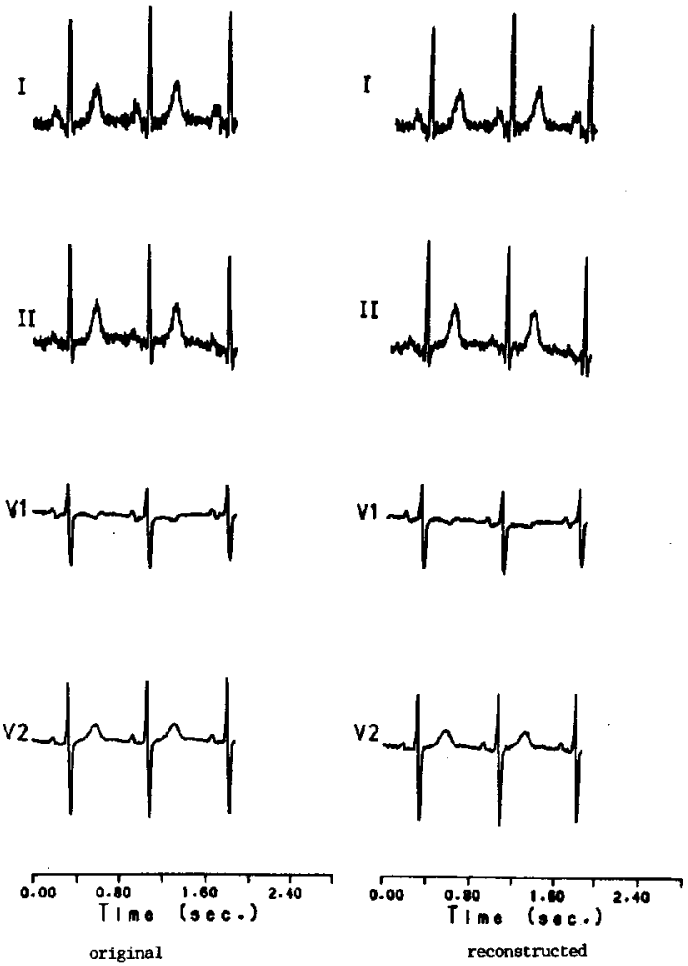

Fig. 6. The original and reconstructed ECG lead signals, I, II, V1, and V2 $(\mathrm{CR}(\mathrm{DCT})=6.17, \mathrm{APRD}=6.19 \%)$.

Decoded time domain signals have a sampling rate which is four times lower than the original sampling rate. Therefore, the decoded signal has to be interpolated by a factor of four.

The digital interpolation is the dual operation of the digital decimation [9]. An interpolator by a factor of two consists of an upsampler which inserts a zero between adjacent samples of its input sequence, and a lowpass filter with a cutoff $\omega=\pi / 2$. To achieve interpolation by a factor of four, we used two successive interpolation by a factor of two blocks as shown in Fig. 5 .

\section{Simulation EXAMPLES}

In this section, the new multichannel ECG compression scheme is compared to the single channel compression scheme of [5]. It 
is very difficult to compare various ECG compression schemes as pointed out in [3] because reported coding results of various ECG data compression schemes are obtained under different recording conditions such as sampling frequency, bandwidth, precision of the sample, and noise level which may drastically effect the currently used performance measures.

Recorded ECG signals are sampled at $500 \mathrm{~Hz}$ with $12 \mathrm{bit} / \mathrm{sample}$ resolution and the line interference is removed by means of an algebraic subtraction technique [11] employing an interference reference channel data. The compression ratio (CR) is defined as follows:

$$
\mathrm{CR}=\frac{N \cdot L \cdot b}{K}
$$

where $N$ is the number of channels, $L$ is the number of samples per channel, $b$ is the resolution of the samples in bits, and $K$ is the total number of bits at the outputs of the coders. Average percent root mean square difference (APRD) [3] is utilized to evaluate the reconstructed signals. The percent root mean square difference (PRD) is defined as follows:

$$
\mathrm{PRD}=\sqrt{\frac{\sum_{n=0}^{N-1}\left[x_{\mathrm{org}}(n)-x_{\mathrm{rec}}(n)\right]^{2}}{\sum_{n=0}^{N-1} x_{\mathrm{org}}^{2}(n)}} \times 100
$$

APRD is the average of the PRD values of the eight ECG channels. In Table I the simulation results are summarized. In the first column of Table I CR values obtained by the SB coder of [5] is given. If DCT (KLT) is used as the linear transformer then the coding results given in second (third) column of the Table I are obtained for given APRD values. Best results are obtained in the third column (KL transform case). Clearly, the new multichannel coding method outperforms the single channel SB coder described in [5]. Original and reconstructed ECG waveforms are shown in Fig. 6 for CR(DCT) $=6.17$ with APRD $=6.19 \%$. The full bandwidth of the ECG signals is considered in the simulation examples summarized in Table I but the line interference had been removed. Presence of line interference would have an adverse effect on compression ratio.

The effect of compressing the data on diagnostic computer analysis results is tested on Cardionics program which is derived from the Mount Sinai program developed by Pordy $e t$ al. in conjunction with CRO-MED Bionics Company [12]. Morphological measurements include intervals, PR, QRS, QT, "width"s, Q, R, S, R', S', T, P, amplitudes, $P, Q, R, R^{\prime}, S, S^{\prime}, T, J J$, and areas of QRS, and QRST. There was no difference in the measurement results of both the compressed and the original data.

Another case is also considered where the raw ECG signals are filtered to attenuate the high frequency noise with a 33-tap equiripple Parks-McClellan FIR filter whose cut-off frequency is equal to 125 Hz. The multichannel ECG compression method is applied to the filtered ECG data. Large increases in the CR values are observed and the coding results are given in Table $\amalg$.

Recently, Hamilton et al. [13] proposed a predictive ECG data compression scheme which subtracts an average beat signal from the original ECG signal before a DPCM type coding. Average beat subtraction can be also utilized in our technique. KL transform of beat subtracted ECG channels may increase the CR value for a fixed APRD value because beat subtracted ECG signals can be considered to be more "stationary" than the raw ECG signals.
TABLE II

CR AND APRD VAlues FOR THE LOW-PASS FILTERed (CUTOFP Frequency $=125 \mathrm{~Hz}$ ) MultichanNel ECG Signals

\begin{tabular}{|c|c|c|c|}
\hline \hline$C R$ of $S B C$ & $C R(D C T)$ & $C R(K L T)$ & $A P R D(\%)$ \\
\hline \hline 4.62 & 5.67 & 7.21 & 3.50 \\
4.66 & 5.76 & 7.61 & 3.71 \\
4.74 & 6.01 & 7.87 & 4.08 \\
4.77 & 6.07 & 8.06 & 4.26 \\
4.90 & 6.11 & 8.55 & 4.70 \\
5.02 & 6.29 & 9.03 & 5.37 \\
5.15 & 6.46 & 9.33 & 5.78 \\
5.22 & 6.48 & 9.41 & 5.94 \\
\hline
\end{tabular}

\section{CONCLUSION}

In this paper, a new multichannel ECG data compression scheme is presented. Simulation examples show that high compression ratios with low APRD values can be achieved. This is because of the fact that, the new technique exploits the inherent correlation among the ECG leads. Recorded ECG signals are decorrelated by using a linear transform which is either an approximate $\mathrm{KL}$ transform or the DCT. The resultant set of uncorrelated signals are coded by using various single channel ECG coding schemes. It is experimentally observed that the multichannel technique produces better results than single channel schemes.

Computational complexity of the multichannel scheme is comparable to single channel ECG coding schemes. The multichannel algorithm can also be implemented by using a digital signal processor for real-time applications, such as transmission of ECG signals over telephone lines.

\section{REFERENCES}

[1] J. Willems, "Common standards for quantitative electrocardiography," J. Med. Eng. Techn., vol. 9, pp. 209-217, 1985.

[2] N. S. Jayant and P. Noll, Digital Coding of Waveforms. Englewood Cliffs, NJ: Prentice-Hall, 1984.

[3] S. M. S. Jalaleddine, C. G. Hutchens, R. D. Strattan, and W. A. Coberly, "ECG data compression techniques-A unified approach," IEEE Trans. Biomed. Eng., vol. BME-37, pp. 329-343, Apr. 1990.

[4] M. Bertrand, R. Guardo, F. A. Roberge, and P. Blondeau, "Microprocessor application for numerical ECG encoding and transmission," Proc. IEEE, vol. 65, pp. 714-722, 1977.

[5] M. C. Aydın, A. E. Çetin, and H. Köymen, "ECG data compression by sub-band coding," Electron. Lett., vol. 27-4, pp. 359-360, 1991.

[6] M. C. Aydin, "Multilead ECG Data Compression by Multirate signal processing and transform domain coding techniques," M.Sc. Thesis, Bilkent University, Ankara, Turkey, 1991.

[7] N. Ahmed, T. J. Natarajan, and K. R. Ran, "Discrete cosine transform," IEEE Trans. Comput., vol. C-23, pp. 90-93, 1974.

[8] N. Ahmed, P. J. Milne, and S. G. Harris, "Electrocardiographic data compression via orthogonal transforms," IEEE Trans. Biomed. Eng., vol BME-22, pp, 484 487, Nov, 1975.

[9] R. E. Crochiere and L. R. Rabiner, "Interpolation and decimation of digital signals-A tutorial review," Proc. IEEE, vol. 69, no. 3, Mar 1981.

[10] C. W. Kim and R. Ansari, "FIR/IIR exact reconstruction filter banks with applications to sub-band coding of images," Midwest Symp. Circuits Syst., CA, 1991.

[11] Y. Z. Ider and H. Koymen, "A new technique for line interference monitoring and reduction in biopotential amplifiers," IEEE Trans. Biomed. Eng., vol. BME-37, pp. 624-631, 1990.

[12] L. Pordy et al., "Computer diagnosis of electrocardiograms: A computer program for contour analysis with classical results of rythm and contour interpretation," Comput. Biomed. Res., vol. 1, pp. 408-433, 1968.

[13] P. S. Hamilton and W. J. Tompkins, "Compression of the ambulatory ECG by average beat subtraction and residual differencing," IEEE Trans. Biomed. Eng., vol. BME-38, pp. 253-260, 1991. 